\title{
The effect of antihistamines on behavioral activity by actigraphy
}

\author{
Tomoyuki Kawada
}

Received: 24 October 2013/Revised: 25 December 2013/Accepted: 2 January 2014/Published online: 10 January 2014

(C) Springer-Verlag Berlin Heidelberg 2014

\section{Dear Editor,}

I have read the article "Effects of single therapeutic doses of promethazine, fexofenadine and olopatadine on psychomotor function and histamine-induced wheal- and flareresponses: a randomized double-blind, placebo-controlled study in healthy volunteers" written by Kamei et al. [1]. The authors clarified the effect of antihistamines on psychomotor function. Although the authors also used wrist actigraphy for behavioral activity, this apparatus could not detect the sedative effects induced by antihistamines. The same authors previously reported a positive findings that olopatadine at $10 \mathrm{mg}$ caused a decrease in behavioral activity as measured by wrist actigraphy [2]. I have two concerns on their report.

As a first concern, there were some errors of description. The authors used a watch-type wrist actigraph, named Actiwatch (Philips Respironics Co Ltd, MA), which can detect two-dimensional movements. In contrast, MiniMotionlogger Actigraph (Ambulatory Monitoring Inc, New York) can detect three-dimensional movements. In addition, the Actiwatch contained a piezoelectric transducer that detects motion in two axes and generates a signal voltage. During each epoch, this apparatus can detect the

T. Kawada $(\bowtie)$

Department of Hygiene and Public Health, Nippon Medical School, 1-1-5 Sendagi, Bunkyo-Ku, Tokyo 113-8602, Japan e-mail: kawada@nms.ac.jp frequency and the intensity of the movements simultaneously. Unfortunately, sleep/wake algorithm by ACTION3 software (Ambulatory Monitoring Inc, New York) could be applied to Mini-Motionlogger Actigraph, not to Actiwatch.

As a second concern, I previously reported the limitation for monitoring wrist movement by Actiwatch [3], by quoting user's manual of Actiwatch. There is a description that the absolute value of activity count from different Actiwatch does not always agree with each other, partly caused by mounting and positioning of this apparatus. I recommend checking reliability of activity count of this apparatus including inter-machine variation for keeping validation of their study.

As the authors conducted their study in young healthy volunteers aged between 18 and 22 years, application of their study outcome is limited only in this generation. Further study is recommended for targeting patients, who need antihistamines to control their clinical symptoms.

Conflict of interest There is no conflict of interest in this study.

\section{References}

1. Kamei H, Isaji A, Noda Y, Ishikawa K, Senzaki K, Yamada K, Sugiura K, Tomita Y, Nabeshima T (2012) Effects of single therapeutic doses of promethazine, fexofenadine and olopatadine on psychomotor function and histamine-induced wheal- and flareresponses: a randomized double-blind, placebo-controlled study in healthy volunteers. Arch Dermatol Res 304(4):263-272

2. Kamei H, Noda Y, Ishikawa K, Senzaki K, Muraoka I, Hasegawa Y, Hindmarch I, Nabeshima T (2003) Comparative study of acute effects of single doses of fexofenadine, olopatadine, D-chlorpheniramine and placebo on psychomotor function in healthy volunteers. Hum Psychopharmacol 18(8):611-618

3. Kawada T (2012) The handling of absolute values of activity counts by actigraphy. J Neurol Sci 312(1-2):194 